\title{
CONTROLE DE PH EM TRATAMENTO DE EFLUENTES UTILIZANDO DIÓXIDO DE CARBONO
}

\author{
E. N. M. SANTOS ${ }^{1}$, M. G. MINATEL ${ }^{1}$, B. F. SANTORO ${ }^{1}$ \\ ${ }^{1}$ Universidade Federal de São Paulo, Departamento de Engenharia Química \\ E-mail para contato: bruno.santoro@unifesp.br
}

\begin{abstract}
RESUMO - O pH é um dos principais parâmetros a controlar no tratamento de efluentes, pois efluentes básicos podem provocar severos danos a fauna e flora dos rios em que forem despejados. Neste trabalho, modela-se uma alternativa à estratégia clássica de neutralização de efluentes usando um ácido forte. Especificamente, considera-se o borbulhamento de gás carbônico $\left(\mathrm{CO}_{2}\right)$ no efluente a ser tratado. O objetivo deste trabalho foi avaliar a performance do sistema de neutralização de um efluente básico através da injeção de $\mathrm{CO}_{2}$, manipulando a pressão parcial do gás através de controladores On/Off e PI. A metodologia consistiu primeiramente na modelagem das reações de neutralização utilizando o software Scilab. Posteriormente, foi feita a síntese dos controladores citados, representando o sistema em malha fechada. As simulações indicaram que o uso de $\mathrm{CO}_{2}$ é uma técnica promissora para a neutralização do sistema, por suas características tamponantes. Comparando os dois controladores, notou-se que o esquema On/Off não foi muito eficiente para o sistema em questão, principalmente na presença de distúrbios, por ter ação menos flexível. O controlador PI adequadamente sintonizado alcançou o setpoint em um tempo menor e rejeitou distúrbios na composição do efluente.
\end{abstract}

\section{INTRODUÇÃO}

$\mathrm{O}$ pH é um dos principais parâmetros a serem controlados no tratamento dos efluentes, já que efluentes básicos despejados sem tratamento em ambientes aquáticos provocam profundos danos ambientais, incluindo a morte de peixes e invertebrado, concentração de metais e precipitação de minerais, principalmente cálcio, que impede a penetração de luz nas águas (GOMES et al., 2015). O modo mais conhecido e utilizado para a regulação do pH de efluentes básicos é pela adição de ácido sulfúrico $\left(\mathrm{H}_{2} \mathrm{SO}_{4}\right)$ no meio, mas esse método possui vários potenciais problemas, como ser difícil de ser manuseado e estocado. Caso o ácido seja dosado incorretamente, pode abaixar excessivamente o $\mathrm{pH}$ do efluente, deixando-o ácido.

Como alternativa ao uso de $\mathrm{H}_{2} \mathrm{SO}_{4}$, pode-se usar o dióxido de carbono $\left(\mathrm{CO}_{2}\right)$ para neutralização de efluentes básicos. Quando dissolvido em água, o $\mathrm{CO}_{2}$ produz uma reação de equilíbrio formando o ácido carbônico (ácido fraco). Esse ácido se ioniza, liberando $\mathrm{H}^{+}$que reage com os íons $\mathrm{OH}^{-}$, neutralizando o efluente. $\mathrm{O}$ uso de $\mathrm{CO}_{2}$ para neutralizar o efluente é sustentável, visto que com seu uso previne-se o acúmulo excessivo de sais como cloratos e 
sulfatos, e sua utilização também é uma forma de reduzir parte da emissão de $\mathrm{CO}_{2}$ para a atmosfera. Por produzir um ácido fraco em contato com a água, o uso de $\mathrm{CO}_{2}$ gera uma menor taxa de corrosão de equipamentos e tubulação, além de seu manuseio ser mais seguro quando comparado ao $\mathrm{H}_{2} \mathrm{SO}_{4}$. Outra vantagem é evitar uma alta acidificação do efluente devido a sua capacidade tamponante (Asti, 2013).

Para controlar o $\mathrm{pH}$ duas formas podem ser utilizadas: controle manual ou automático (utilizando controladores). Processos manuais geralmente estão sujeitos a erros humanos e são difíceis de serem padronizados. Já com a utilização de controladores tem-se um ganho de produtividade com o cálculo automático da quantidade de produto químico que deve ser utilizado (Stephanopoulos, 1984). Outra vantagem está no fato que variações mínimas de pH são suficientes para induzir o controlador a liberar uma quantidade adequada de produto necessário para trabalhar na faixa estabelecida.

Apesar das vantagens, o controle automático de $\mathrm{pH}$ é muito difícil devido a sua característica não-linear, provocando variações bruscas na ação de controle. Desse modo, um controlador mal sintonizado pode ter problemas de estabilidade e oscilações nas variáveis de processo, o que pode causar mais prejuízos do que a utilização de um simples controle manual (Stephanopoulos, 1984).

O objetivo deste trabalho foi modelar a reação de neutralização de um efluente básico utilizando $\mathrm{CO}_{2}$ no Scilab/Xcos, e realizar a síntese de controladores On/Off e PI para controle do $\mathrm{pH}$ desse efluente, comparando a eficiência das ações de controle e a complexidade da síntese dos arranjos de controle.

\section{MODELAGEM DO SISTEMA}

No processo de neutralização de um efluente básico, primeiro tem-se a adição de $\mathrm{CO}_{2}$ gasoso no sistema, sendo o mesmo absorvido para obtenção do $\mathrm{CO}_{2}$ aquoso como mostra a Equação 1 (Ámez, 2013):

$$
\mathrm{CO}_{2(g)} \rightleftharpoons \mathrm{CO}_{2(a q)}
$$

Os balanços das espécies de interesse, com as constantes cinéticas apropriadas, foram extraídos de Schulz et al. (2006), contemplando as seguintes reações:

$$
\begin{aligned}
& \mathrm{CO}_{2(a q)}+\mathrm{H}_{2} \mathrm{O} \underset{k-1}{\stackrel{k+1}{\rightleftarrows}} \mathrm{HCO}_{3}^{-}+\mathrm{H}^{+} \\
& \mathrm{CO}_{2(a q)}+\mathrm{OH}^{-} \underset{k-4}{\stackrel{k+4}{\rightleftarrows}} \mathrm{HCO}_{3}^{-} \\
& \mathrm{H}_{2} \mathrm{O} \underset{k-6}{\stackrel{k+6}{\rightleftarrows}} \mathrm{H}^{+}+\mathrm{OH}^{-} \\
& \mathrm{HCO}_{3}^{-}+\mathrm{OH}^{-} \underset{k_{-5}^{O H^{-}}}{\stackrel{k_{+5}^{O H^{-}}}{\rightleftarrows}} \mathrm{CO}_{3}^{2-}+\mathrm{H}_{2} \mathrm{O} \\
& \mathrm{CO}_{3}{ }^{2-}+\mathrm{H}^{+} \underset{k_{-5}^{H^{+}}}{\stackrel{k_{+5}^{H^{+}}}{\rightleftarrows}} \mathrm{HCO}_{3}^{-}
\end{aligned}
$$




\section{Congresso Brasileiro de Engenharia Química \\ em Iniciação Científica \\ UFSCar - São Carlos - SP

Todas as reações são reversíveis e os valores das constantes cinéticas diretas (Representadas por subscrito “+”) e inversas (Representadas por subscrito "-"). A partir da reação do dióxido de carbono aquoso com a água, forma-se o ácido carbônico (fraco), liberando os íons $\mathrm{HCO}_{3}{ }^{-}$e $\mathrm{H}^{+}$com uma constante de equilíbrio $\mathrm{k}_{1}$. $\mathrm{O} \mathrm{CO}_{2}$ ainda reage com os íons hidroxila presentes na solução inicial, formando também íons $\mathrm{HCO}_{3}{ }^{-}$sendo a cinética dada pela constante de equilíbrio $\mathrm{k}_{4}$ (Equação 3). A dissociação da água também ocorre, sendo regida pela constante $\mathrm{k}_{6}$ (Equação 4).

$\mathrm{O}$ ânion $\mathrm{HCO}_{3}{ }^{-}$reage com os íons hidroxila conforme dinâmica definida pela constante $\mathrm{k}_{5}{ }^{\mathrm{OH}-}$ (Equação 5) gerando íons $\mathrm{CO}_{3}{ }^{-2}$ e água. Os ânions carbonato formados reagem com o cátion hidrogênio presente na solução, formando novamente o ânion de bicarbonato, sendo a cinética dada pela constante $\mathrm{k}_{5}{ }^{\mathrm{H}+}$ (Equação 6). Os valores utilizados foram (SCHULZ et al., 2006):

$\mathbf{k}_{+\mathbf{1}}=3,71 \times 10^{-2} \mathrm{~s}^{-1} ; \mathbf{k}_{-\mathbf{1}}=2,67 \times 10^{4} \mathrm{~kg} \mathrm{~mol}^{-1} \mathrm{~s}^{-1} ; \mathbf{k}_{+\mathbf{4}}=2,23 \times 10^{3} \mathrm{~kg} \mathrm{~mol}^{-1} \mathrm{~s}^{-1} ; \mathbf{k}_{\mathbf{- 4}}=9,71 \times 10^{-}$ ${ }^{5} \mathrm{~s}^{-1} ; \mathbf{k}+{ }_{5}{ }^{\mathbf{H +}}=5,00 \times 10^{10} \mathrm{~kg} \mathrm{~mol}^{-1} \mathrm{~s}^{-1} ; \mathbf{k}_{-5}{ }^{\mathbf{H +}}=59,44 \mathrm{~s}^{-1} ; \mathbf{k}+5{ }^{\mathbf{O H}-}=6,00 \times 10^{9} \mathrm{~kg} \mathrm{~mol}^{-1} \mathrm{~s}^{-1} ; \mathbf{k}-$ ${ }_{\mathbf{5}}^{\mathbf{O H}^{-}}=3,06 \times 10^{5} \mathrm{~s}^{-1} ; \mathbf{k}_{+\mathbf{6}}=1,40 \times 10^{-3} \mathrm{~mol} \mathrm{~kg}^{-1} \mathrm{~s}^{-1} ; \mathbf{k}_{\mathbf{- 6}}=1,40 \times 10^{11} \mathrm{~mol}^{-1} \mathrm{~kg} \mathrm{~s}^{-1}$

Para obter a concentração de $\mathrm{CO}_{2}$ aquoso é necessário considerar a dissolução do $\mathrm{CO}_{2}$ gasoso em água. Essa concentração pode ser obtida aplicando-se a Lei de Henry que modela essa dissolução. A Equação 7 abaixo mostra a Lei de Henry (SHAPLEY, 2011).

$$
K_{H}=\frac{p^{i}}{\left[g s_{(a q)}\right]}
$$

Em que $\mathrm{K}_{\mathrm{H}}=$ Constante da Lei de Henry para o gás trabalhado; [gás ${ }_{(\mathrm{aq})}$ ] = Concentração do gás dissolvido; $\mathrm{P}^{\mathrm{i}}=$ Pressão de um gás puro, ou pressão parcial de um gás se existe uma mistura de gases na solução. De acordo com Shapley (2011), o valor de $\mathrm{K}_{\mathrm{H}}$ para o CO2 é de 29,41 atm.L.mol ${ }^{-1}$ a $25^{\circ} \mathrm{C}$.

Para fins de simulação, foi considerado um tanque com volume de $1000 \mathrm{~L}$, um fluxo de entrada constante $\left(15 \mathrm{~m}^{3} \cdot \mathrm{h}^{-1}\right.$ ou $4,15 \mathrm{~L} \cdot \mathrm{s}^{-1}$ ) de um efluente básico (que teve a composição simplificada para água e uma determinada concentração de $\mathrm{OH}^{-}$para cada $\mathrm{pH}$ analisado) e um fluxo constante de saída do efluente neutralizado com valor igual ao de entrada. Admitiu-se também que a densidade do efluente era de $1 \mathrm{~kg}$. $\mathrm{L}^{-1}$ e que a temperatura era constante a $25^{\circ} \mathrm{C}$.

Foi utilizado o Scilab/Xcos (SCILAB ENTERPRISES, 2012) a fim de simular as equações diferenciais relativas aos balanços molares por componente do sistema, além de reportar os gráficos e resultados para análises e discussões. Foi definido também que o pH seria a variável controlada e a pressão parcial de $\mathrm{CO}_{2}$ a variável manipulada.

\section{CONTROLADORES}

Controladores PID são amplamente utilizados na indústria devido a sua simplicidade e eficiência. $\mathrm{O}$ ajuste de controle das variáveis é contínuo, realizado a partir da diferença entre o setpoint da variável controlada e o valor do sinal de saída. O controlador PID possui três 
parâmetros que influenciam em sua ação: Proporcional, Integral e Derivativo. O controlador PID é implementado em Xcos na forma dada pela equação 8 a seguir:

$$
\mathrm{u}^{\prime}(\mathrm{t})=\text { P.e } \mathrm{e}(\mathrm{t})+\mathbf{I} \cdot \int_{0}^{\mathrm{t}} \mathrm{e}(\mathrm{t}) \mathrm{dt}+\mathbf{D} \frac{\mathrm{de}(\mathrm{t})}{\mathrm{dt}}
$$

No caso presente, apenas os modos Proporcional e Integral foram utilizados nas simulações. A introdução do modo derivativo causou problemas numéricos durante a resolução das equações diferenciais e portanto as simulações consideraram apenas o caso PI (Proporcional e Integral). O controlador On/Off foi construído a partir dos blocos fundamentais disponíveis no Xcos. O bloco obtido permite que o usuário configure o valor de erro que aciona o controlador e as suas saídas nos estados ligado e desligado.

\section{RESULTADOS E DISCUSSÕES}

Feita a modelagem do sistema e a síntese dos controladores, pôde-se simular a neutralização do sistema utilizando os controladores On/Off e PI. O pH foi definido como a variável controlada e a pressão parcial de $\mathrm{CO}_{2}$ a variável manipulada. Através de simulações em malha aberta, determinou-se que o valor de pressão parcial de $\mathrm{CO}_{2}$ que deixava o sistema com $\mathrm{pH}$ próximo a 7 era de $3,48 \times 10^{-3} \mathrm{~atm}$.

\subsection{Controlador On/Off}

Para o controlador On/Off foi realizada uma simulação com pH inicial em 10 e utilizouse um valor máximo de 4,00 × $10^{-3}$ atm e mínimo de $3,40 \times 10^{-3}$ atm de pressão parcial de $\mathrm{CO}_{2}$. $\mathrm{O}$ valor da pressão máxima foi escolhido por estar perto do valor de pressão obtido em malha aberta como ideal. A pressão mínima escolhida tinha valor muito próximo da encontrada em malha aberta, visando uma baixa alternância entre o "liga e desliga" e consequentemente um menor desgaste do atuador, além de uma estabilização do $\mathrm{pH}$ na faixa de neutralidade aceitável, entre 6,5 e 7,5, como indicado nas Figuras 1 e 2. Notou-se que o sistema conseguiu estabilizar o $\mathrm{pH}$ na faixa de neutralidade desejada e sem muitas alternâncias do controlador.

Figura 1: $\mathrm{pH}$, controle On/Off

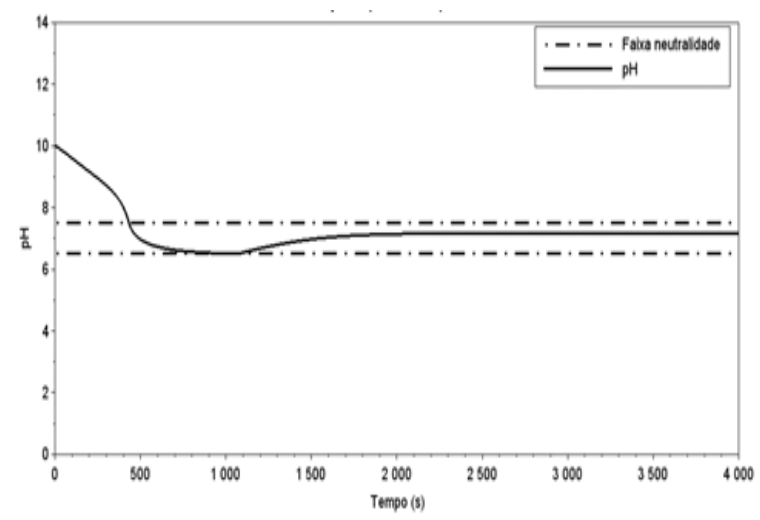

Figura 2: pressão de $\mathrm{CO}_{2}$, controle On/Off

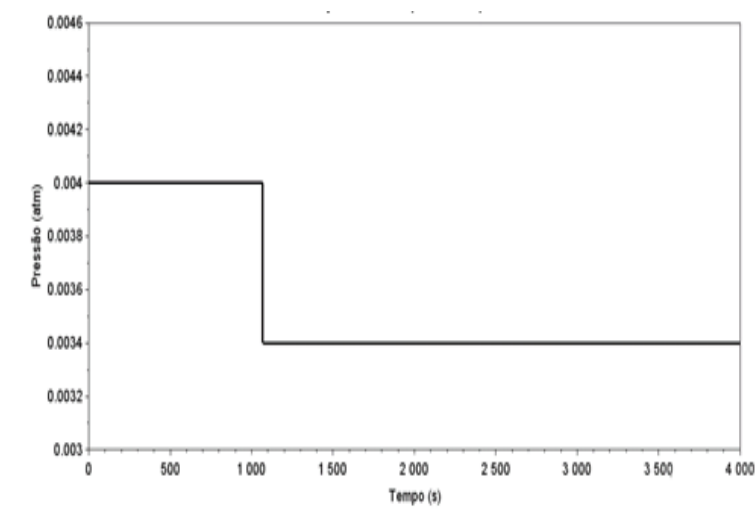




\section{Congresso Brasileiro de Engenharia Química em Iniciação Científica UFSCar - São Carlos - SP 16 a 19 de Julho de 2017}

\subsection{Controlador PI}

Pelo estudo do sistema em malha aberta, percebeu-se que o $\mathrm{pH}$ diminuía com o aumento de pressão de $\mathrm{CO}_{2}$, então se pode afirmar que o ganho do processo é negativo. Tendo isso em vista, o ganho do controlador também precisa ser negativo, e então $\mathrm{P}$ e I devem ser negativos para o controlador conseguir levar o sistema ao setpoint de forma estável. Sintonizando por tentativa e erro, encontraram-se parâmetros aceitáveis iguais a $\mathrm{P}=-710^{-3}$ e I $=-310^{-5}$. As Figuras 3 e 4 a seguir mostram respectivamente a variação do $\mathrm{pH}$ e da pressão:

Figura 3: pH, controle PI

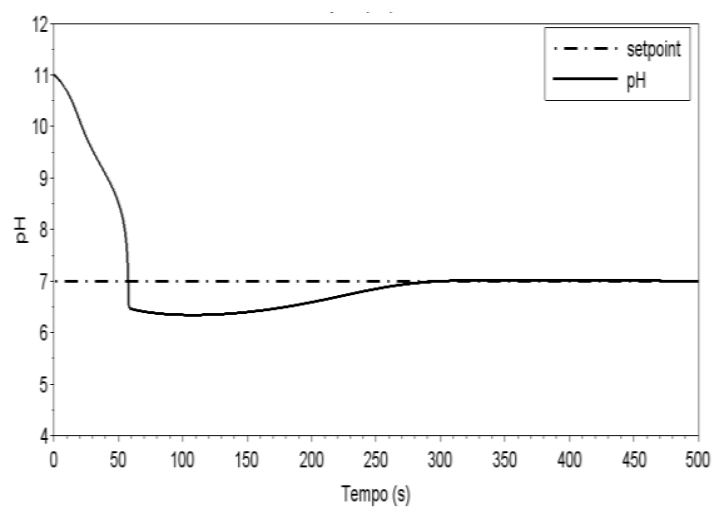

Figura 4: pressão de $\mathrm{CO}_{2}$, controle On/Off

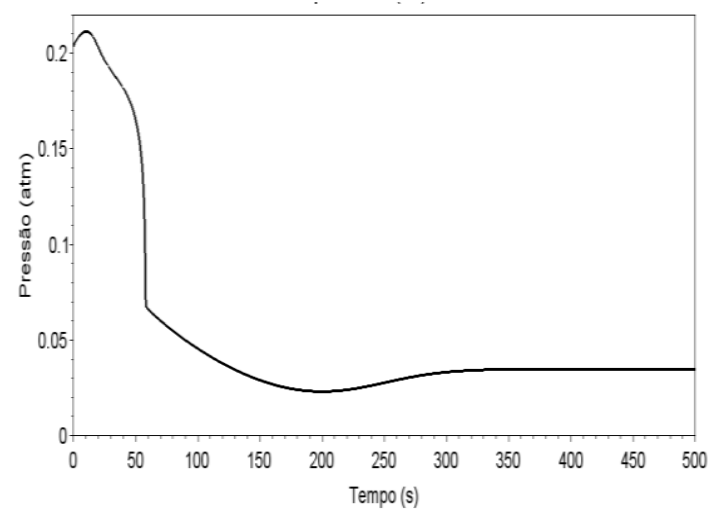

Para comparar os controladores, uma das simulações realizadas considerou o sistema com um distúrbio aumentando a concentração de entrada de $\mathrm{OH}^{-}$de $1,010^{-4} \mathrm{~mol} / \mathrm{L}$ para 1,5 $10^{-4} \mathrm{~mol} / \mathrm{L}$ no instante $\mathrm{T}=1900 \mathrm{~s}$. Os resultados estão apresentados nas Figuras 5 e 6 :

Figura 5: $\mathrm{pH}$, comparação entre Figura 6: pressão de $\mathrm{CO}$ 2, comparação controladores entre controladores
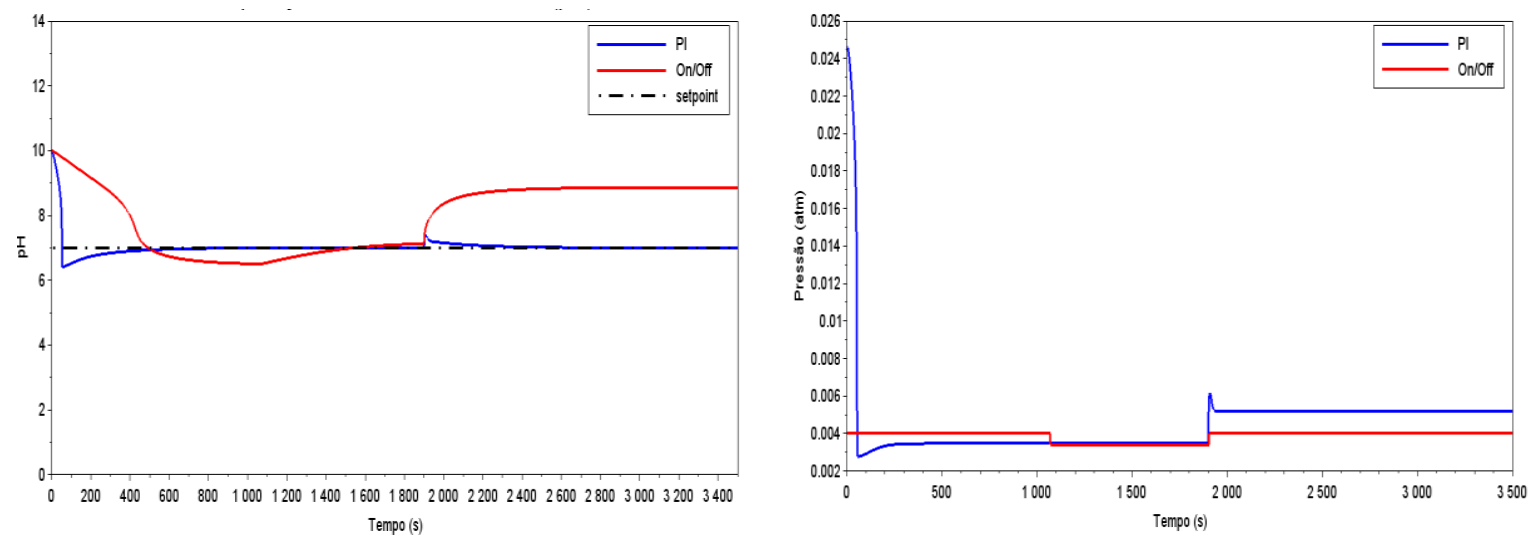

O controlador PI alcança o setpoint mais rápido inicialmente e consegue manter o sistema no valor desejado após o distúrbio, enquanto o On/Off deixa a entrada saturada em um valor não adequado. Se os limites mínimo e máximo da pressão de $\mathrm{CO}_{2}$ fossem alargados no controlador On/Off, a performance do sistema seria ainda pior, com chaveamento 
constante entre os estados. Dessa forma, não foi possível obter uma performance adequada para esse controlador no caso de um distúrbio persistente.

\section{Conclusões}

A modelagem da reação de neutralização utilizando o $\mathrm{CO}_{2}$ no Scilab/Xcos foi considerada satisfatória por apresentar o comportamento esperado para as curvas de neutralização. Graças à capacidade tamponante do sistema, observaram-se quedas menos bruscas no $\mathrm{pH}$ ao variar a pressão parcial de $\mathrm{CO}_{2}$, em comparação com adição de ácido forte.

Comparando a performance dos dois controladores, notou-se que o controlador On/Off não foi muito eficiente para um sistema de controle de $\mathrm{pH}$, principalmente quando há distúrbios no sistema. Já o controlador PI apresentou bons resultados no controle do $\mathrm{pH}$ alcançando o setpoint em um tempo rápido e se comportando bem na presença de distúrbios de diferentes intensidades, sendo o controlador indicado para este tipo de controle.

A continuação desse trabalho envolve melhorias na modelagem e no controle. $O$ primeiro ponto pode ser aperfeiçoado considerando-se uma composição mais detalhada do efluente a ser tratado, com eventuais interações adicionais com o $\mathrm{CO}_{2}$ borbulhado. Do ponto de vista de controle, a plataforma Scilab se mostrou pouco robusta para um problema dessas dimensões; em particular, a inclusão do modo derivativo no controlador causava o algoritmo de resolução de equações diferenciais a falhar. Uma alternativa é buscar outro ambiente de programação, como Matlab (através do módulo Simulink) ou Octave, para manter o caráter de código livre.

\section{REFERÊNCIAS}

ÁMEZ, P. G. Estudo da absorção de $\mathrm{CO}_{2}$ de gases exaustos de usinas termelétricas em reator cilíndrico contendo solução de $\mathrm{NaOH}$. Universidade Federal do Rio de Janeiro. Rio de Janeiro. 2013.

ASTI. Carbon Dioxide for pH Control. Site da Asti sensor, 2013. Disponível em: $<\mathrm{http} / / / \mathrm{www}$.astisensor.com/Carbon_Dioxide_Versus_Mineral_Acids.pdf $>$. Acesso em: 29 ago. 2016.

GOMES, H.; MAYES, W.; ROGERSON, M.; STEWART, D.I.; BURKE, I.T. Alkaline residues and the environment: a review of impacts, management practices and opportunities. J. Clean. Prod. v. 112, p. 1-12, 2015.

SCILAB ENTERPRISES (2012). Scilab: Free and Open Source software for numerical computation (OS, Version 5.5.2) [Software]. Disponível em: http://www.scilab.org.

SHAPLEY, P. University of Illinois, 2011. Disponível em:

<http://butane.chem.uiuc.edu/pshapley/GenChem1/L23/web-L23.pdf>. Acesso em:07 set. 2016.

SCHULZ, K. G.; RIEBESELL, U.; ROST, B.; THOMS, S.; ZEEBE, R. E Determination of the rate constants for the carbon dioxide to bicarbonate inter-conversion in $\mathrm{pH}$-buffered seawater systems. Marit. Chem., n. 100, p. 53-65, 2006.

STEPHANOPOULOS, G. Chemical process control. New Jersey: Prentice hall, 1984. 